\title{
A Simple Approach to Constructing Quasi-Sudoku-based Sliced Space-Filling Designs
}

\author{
Diane Donovan ${ }^{1}$, Benjamin Haaland ${ }^{2,3}$, David J. Nott ${ }^{4}$ \\ ${ }^{1}$ University of Queensland, Brisbane, Australia \\ ${ }^{2}$ Georgia Institute of Technology, USA \\ ${ }^{3}$ Duke-NUS Graduate Medical School, Singapore \\ ${ }^{3}$ National University of Singapore, Singapore
}

October 11, 2018

\begin{abstract}
Sliced Sudoku-based space-filling designs and, more generally, quasi-sliced orthogonal array-based space-filling designs are useful experimental designs in several contexts, including computer experiments with categorical in addition to quantitative inputs and cross-validation. Here, we provide a straightforward construction of doubly orthogonal quasi-Sudoku Latin squares which can be used to generate sliced space-filling designs which achieve uniformity in one and two-dimensional projections for both the full design and each slice. A construction of quasi-sliced orthogonal arrays based on these constructed doubly orthogonal quasi-Sudoku Latin squares is also provided and can, in turn, be used to generate sliced space-filling designs which achieve uniformity in one and two-dimensional projections for the full design and and uniformity in two-dimensional projections for each slice. These constructions are very practical to implement and yield a spectrum of design sizes and numbers of factors not currently broadly available.
\end{abstract}

KEY WORDS: Computer experiments, space-filling designs, Sudoku, sliced experimental designs. 


\section{Introduction}

In the popular game Sudoku, players are presented with a nine-by-nine array, divided into nine three-by-three subsubarrays, and partially filled with the numbers 1 through 9 . The goal is to fill the nine-by-nine array with the numbers 1 through 9 so that each row, column, and three-by-three subarray contains no repeated numbers. See a starting and completed Sudoku square in Figure 1 [8].

\begin{tabular}{|lll|lll|lll|}
\hline & & & 7 & 3 & 5 & 8 & 2 & \\
8 & & & & 1 & & & & 5 \\
& & & & 2 & 6 & & 1 & \\
\hline & 8 & 1 & & 7 & & & & 9 \\
& 6 & & & 9 & & & 5 & \\
9 & & & & 5 & & 3 & 8 & \\
\hline & 3 & & 1 & 4 & & & & \\
4 & & & & 6 & & & & 3 \\
& 9 & 2 & 3 & 8 & 7 & & & \\
\hline
\end{tabular}

\begin{tabular}{|lll|lll|lll|}
\hline 1 & 4 & 9 & $\mathbf{7}$ & $\mathbf{3}$ & $\mathbf{5}$ & $\mathbf{8}$ & $\mathbf{2}$ & 6 \\
$\mathbf{8}$ & 2 & 6 & 9 & $\mathbf{1}$ & 4 & 7 & 3 & $\mathbf{5}$ \\
3 & 5 & 7 & 8 & $\mathbf{2}$ & $\mathbf{6}$ & 9 & $\mathbf{1}$ & 4 \\
\hline 5 & $\mathbf{8}$ & $\mathbf{1}$ & 2 & $\mathbf{7}$ & 3 & 4 & 6 & $\mathbf{9}$ \\
2 & $\mathbf{6}$ & 3 & 4 & $\mathbf{9}$ & 8 & 1 & $\mathbf{5}$ & 7 \\
$\mathbf{9}$ & 7 & 4 & 6 & $\mathbf{5}$ & 1 & $\mathbf{3}$ & $\mathbf{8}$ & 2 \\
\hline 7 & $\mathbf{3}$ & 5 & $\mathbf{1}$ & $\mathbf{4}$ & 2 & 6 & 9 & 8 \\
$\mathbf{4}$ & 1 & 8 & 5 & $\mathbf{6}$ & 9 & 2 & 7 & $\mathbf{3}$ \\
6 & $\mathbf{9}$ & $\mathbf{2}$ & $\mathbf{3}$ & $\mathbf{8}$ & $\mathbf{7}$ & 5 & 4 & 1 \\
\hline
\end{tabular}

Figure 1: A starting and completed Sudoku square [8].

Sets of (completed) Sudoku squares, as well as generalizations thereof, can be used to construct sliced space-filling designs achieving maximal uniformity in both one and twodimensional projections for both the complete design and each subdesign, or slice. Sliced space-filling designs are experimental designs which can be partitioned into groups of subdesigns so that both the full design and each subdesign achieve some type of uniformity. These types of designs are broadly useful for collecting data from computer experiments, large and time-consuming mathematical codes used to model real world systems such as the climate or a component in an engineering design problem. Sliced space-filling designs are particularly useful for computer experiments with qualitative and quantitative inputs [5], multiple levels of accuracy [3], and cross-validation problems in the context of computer experiments [10]. Sudoku-based sliced space-filling designs were introduced in [9] and a construction was given using doubly orthogonal Sudoku squares, whose complete arrays are orthogonal and whose subarrays are orthogonal after a projection. In [9], doubly orthogonal Sudoku squares were constructed using the techniques developed in [4] along with a subfield projection.

Here, we give a straightforward construction of doubly orthogonal quasi-Sudoku squares relying on the existence of sets of orthogonal Latin squares, which are relatively welldescribed and available for a broad range of sizes [1, 2, 6]. Further, the presented construction of sliced Sudoku-based space-filling designs is available for a spectrum of sample 
sizes and number of factors combinations. The remainder of this article is organized as follows. Section 2 provides notation and definitions which will be used throughout. Section 3 provides a construction for sets of pairwise doubly orthogonal quasi-Sudoku Latin squares which is based on sets of orthogonal Latin squares. Section 4 illustrates the presented techniques with an example. Section 5 notes the connection with quasi-sliced asymmetric orthogonal arrays. Finally, Section 6 reviews the construction of Sudoku-based sliced space-filling designs from [9].

\section{Notation and Definitions}

Let $[n]=\{0,1, \ldots, n-1\}$ and $A=[A(i, j)]$ be a Latin square of order $n$; that is, an $n \times n$ array in which each of the entries in a set $N$ (usually $[n]$ ) of size $n$ occurs once in every row and once in every column. Two Latin squares $A=[A(i, j)]$ and $B=[B(i, j)]$, of the same order, are said to be orthogonal if, when we superimpose one on top of the other, the arrays contain each of the $n^{2}$ ordered pairs $(x, y), x, y \in N$ exactly once. It is useful to note that $A$ and $B$ are orthogonal if and only if for all $p, q, s, t \in N$

$$
A(p, q)=A(s, t) \Longrightarrow B(p, q) \neq B(s, t)
$$

Let $A=[A(i, j)]$ and $B=[B(i, j)]$ be two orthogonal Latin squares of order $n$. For $n=s^{2}$, let $\Pi$ denote a projection from $N$ to $[s], \Pi: N \rightarrow[s]$, and let $\mathcal{O}=\{(\Pi(A(i, j)), \Pi(B(i, j))) \mid$ $i, j \in N\}$. We may think of $\mathcal{O}$ as an $n \times n$ array obtained by superimposing $\Pi(A)$ and $\Pi(B)$. The Latin squares $A$ and $B$ are said to be doubly orthogonal if there exists a projection $\Pi$ such that $\mathcal{O}$ can be partitioned into $s \times s$ subarrays with the cells of each subarray containing the $s^{2}$ ordered pairs $(x, y), 0 \leqslant x, y \leqslant s-1$. For $n=r s$, let $\Pi_{r}$ denote a projection from $N$ to $[r], \Pi_{r}: N \rightarrow[r]$, let $\Pi_{s}$ denote a projection from $N$ to $[s], \Pi_{s}: N \rightarrow[s]$, and $\mathcal{O}=\left\{\left(\Pi_{r}(A(i, j)), \Pi_{s}(B(i, j))\right) \mid i, j \in N\right\}$. The Latin squares $A$ and $B$ are said to be doubly orthogonal if there exist projections $\Pi_{r}$ and $\Pi_{s}$ such that $\mathcal{O}$ can be partitioned into $r \times s$ subarrays with the cells of each subarray containing the $r s$ ordered pairs $(x, y)$ where $0 \leqslant x \leqslant r-1$ and $0 \leqslant y \leqslant s-1$.

An $m^{2} \times m^{2}$ array is said to be a Sudoku Latin square, on the set $X$ of size $m^{2}$, if it is a Latin square and we can label the rows by $(p, s), 0 \leqslant p, s \leqslant m-1$ and columns by $(q, t), 0 \leqslant q, t \leqslant m-1$ such that for each $p$ and $q$ the subarray defined by the cells

$$
((p, s),(q, t)), \quad 0 \leqslant s, t \leqslant m-1
$$

contains each entry of $X$ precisely once. A $m n \times m n$ array is said be a quasi-Sudoku Latin square, on the set $X$ of order $m n$, if it is a Latin square and we can label the rows by 
$(p, s), 0 \leqslant p \leqslant m-1,0 \leqslant s \leqslant n-1$ and columns $(q, t), 0 \leqslant q \leqslant n-1,0 \leqslant t \leqslant m-1$ such that for each $p$ and $q$ the subarray defined by the cells

$$
((p, s),(q, t)), \quad 0 \leqslant s \leqslant n-1, \quad 0 \leqslant t \leqslant m-1,
$$

contains each entry of $X$ precisely once. Two Sudoku Latin squares or quasi-Sudoku Latin squares are orthogonal (doubly orthogonal) if they are orthogonal (doubly orthogonal) Latin squares.

\section{Construction of Doubly Orthogonal Quasi-Sudoku Latin Squares}

Here, we construct quasi-Sudoku Latin squares which are doubly orthogonal using sets of (pairwise) orthogonal Latin squares. This is done by first using a direct product construction to construct orthogonal quasi-Sudoku Latin squares and then showing that they are doubly orthogonal.

For the remainder of this section we will take $A_{1}$ and $A_{2}$ to be two Latin squares of order $m$ and $n$, respectively. We can construct a new Latin square of order $m n$ by taking the direct product, $A_{1} \otimes A_{2}$, of $A_{1}$ with $A_{2}$, where $\left(A_{1}(p, q), A_{2}(s, t)\right)$ is the element in row $n p+s$ and column $n q+t$ of $A_{1} \otimes A_{2}, 0 \leqslant p, q \leqslant m-1$ and $0 \leqslant s, t \leqslant n-1$. Label the rows of $A_{1} \otimes A_{2}$ as $(p, s)$ and the columns of $A_{1} \otimes A_{2}$ as $(q, t)$. For fixed $p$ and $q$, the subarray defined by the set of cells $\{((p, s),(q, t)) \mid 0 \leqslant s, t \leqslant n-1\}$ is then isomorphic to $A_{2}$.

The next proposition attests that orthogonality is maintained under the direct product. For a proof, see Dénes and Keedwell [2] page 427.

Proposition 3.1. If $A_{1}$ and $B_{1}$ are orthogonal Latin squares of order $m$ and $A_{2}$ and $B_{2}$ are orthogonal Latin squares of order $n$, then $A_{1} \otimes A_{2}$ and $B_{1} \otimes B_{2}$ are orthogonal Latin squares of order mn.

An example of orthogonal Latin squares $A_{1} \otimes A_{2}$ and $B_{1} \otimes B_{2}$, constructed using the orthogonal Latin squares shown in Figure 2 , is described in Section 4 and shown in Figure 3.

Proposition 3.2. If $A_{1}$ and $B_{1}$ are orthogonal Latin squares of order $m$ and $A_{2}$ and $B_{2}$ are orthogonal Latin squares of order $n$, then $A_{1} \otimes A_{2}$ and $B_{1} \otimes B_{2}$ are doubly orthogonal quasi-Sudoku Latin squares of order mn. 
Proof. We begin by verifying that there exists an arrangement of the rows of both arrays $A_{1} \otimes A_{2}$ and $B_{1} \otimes B_{2}$ which verifies that they are quasi-Sudoku latin squares.

For fixed $q$, the entries $A_{1}(p, q)$ and $B_{1}(p, q)$ take the values in column $q$ of $A_{1}$ and $B_{1}$ respectively. That is, for fixed $q,\left\{A_{1}(p, q) \mid 0 \leqslant p \leqslant m-1\right\}=\left\{B_{1}(p, q) \mid 0 \leqslant p \leqslant\right.$ $m-1\}=[m]$. Likewise, for fixed $s$, the entries $A_{2}(s, t)$ and $B_{2}(s, t)$ take values in row $s$ of $A_{2}$ and $B_{2}$, respectively. That is, for fixed $s,\left\{A_{2}(s, t) \mid 0 \leqslant t \leqslant n-1\right\}=\left\{B_{2}(s, t) \mid\right.$ $0 \leqslant t \leqslant n-1\}=[n]$. Thus for fixed $q$ and $s$

$$
\begin{aligned}
{[m] \times[n] } & =\left\{\left(A_{1}(p, q), A_{2}(s, t)\right) \mid 0 \leqslant p \leqslant m-1,0 \leqslant t \leqslant n-1\right\} \\
& =\left\{\left(B_{1}(p, q), B_{2}(s, t)\right) \mid 0 \leqslant p \leqslant m-1,0 \leqslant t \leqslant n-1\right\} .
\end{aligned}
$$

Hence we will assume that the rows of $A_{1} \otimes A_{2}$ and $B_{1} \otimes B_{2}$ have been reordered by moving row $n p+s$ to position $m s+p$, where $0 \leqslant p \leqslant m-1$ and $0 \leqslant s \leqslant n-1$. The order of the columns, however, remains as $q n+t$, where $0 \leqslant q \leqslant m-1$ and $0 \leqslant t \leqslant n-1$. Now for fixed $s$ and $q$ the subarrays of $A_{1} \otimes A_{2}$ and $B_{1} \otimes B_{2}$ defined by the intersection of rows $m s+p$ with columns $q n+t$, where $0 \leqslant p \leqslant m-1$ and $0 \leqslant t \leqslant n-1$ contains each of the $m n$ entries precisely once. Note that the reordering of rows has been consistently applied to both $A_{1} \otimes A_{2}$ and $B_{1} \otimes B_{2}$, therefore these Latin squares are still orthogonal.

We select two onto functions $\Pi_{m}:[m] \times[n] \rightarrow[m]$ and $\Pi_{n}:[m] \times[n] \rightarrow[n]$ such that if $\Pi_{m}\left(A_{1}(p, q), A_{2}(s, t)\right)=\Pi_{m}\left(A_{1}(p, q), A_{2}\left(s, t^{\prime}\right)\right)$ then $t \neq t^{\prime}$ and if $\Pi_{n}\left(B_{1}(p, q), B_{2}(s, t)\right)=$ $\Pi_{n}\left(B_{1}(p, q), B_{2}\left(s, t^{\prime}\right)\right)$ then $t=t^{\prime}$. Such functions are not hard to find for instance we could take

$$
\begin{aligned}
\Pi_{m}\left(A_{1}(p, q), A_{2}(s, t)\right) & =A_{1}(p, q) \text { and } \\
\Pi_{n}\left(B_{1}(p, q), B_{2}(s, t)\right) & =B_{2}(s, t)
\end{aligned}
$$

or if $m$ and $n$ are coprime, with $m>n$, take

$$
\begin{aligned}
\Pi_{m}\left(A_{1}(p, q), A_{2}(s, t)\right) & =\left(n \times A_{1}(p, q)+A_{2}(s, t)\right) \bmod m \text { and } \\
\Pi_{n}\left(B_{1}(p, q), B_{2}(s, t)\right) & =\left(n \times B_{1}(p, q)+B_{2}(s, t)\right) \bmod n .
\end{aligned}
$$

In this latter case since $m$ and $n$ are coprime and $m>n$, for fixed $q,\left\{n \times A_{1}(p, q) \bmod m \mid\right.$ $0 \leqslant p \leqslant m-1\}=[m]$ and since $A_{2}(s, t)<m,\left\{n \times A_{1}(p, q)+A_{2}(s, t) \bmod m \mid 0 \leqslant p \leqslant\right.$ $m-1\}=[m]$, so for any fixed $q, s$ and $t,\left\{\Pi_{m}\left(\left(A_{1}(p, q), A_{2}(s, t)\right)\right) \mid 0 \leqslant p \leqslant m-1\right\}=[m]$. Further, $\left\{\left(\Pi_{m}\left(\left(A_{1}(p, q), A_{2}(s, t)\right)\right),\left(\Pi_{n}\left(\left(B_{1}(p, q), B_{2}(s, t)\right)\right) \mid 0 \leqslant p \leqslant m-1,0 \leqslant t \leqslant n\right\}=\right.\right.$ $[m] \times[n]$. This verifies that $A_{1} \otimes A_{2}$ and $B_{1} \otimes B_{2}$ are doubly orthogonal quasi-Sudoku squares. 
It should be noted that the different projections given in the above proof produce non-isomorphic squares. This will be illustrated in the example given below. For $m=n$, the extension of these pairwise properties to more than two orthogonal direct product designs is immediate, if the component designs are available. Further, it should be noted that when we write $A_{1} \otimes A_{2}$ and $B_{1} \otimes B_{2}$ are orthogonal quasi-Sudoku Latin squares we are assuming that the rows of the direct products have been rearranged to the required format for Sudoku Latin squares.

\section{An Example}

As an illustration, we will construct doubly orthogonal quasi-Sudoku Latin squares of order 12. We begin with two orthogonal Latin squares of order $n=3$ and two of the three orthogonal Latin squares of order $m=4$, as shown in Figure 2, Note it does not matter which two orthogonal Latin squares of order 4 we choose, so we arbitrarily select the first and the last. In general, if the underlying Latin squares are non-isomorphic then it is possible to construct sets of non-isomorphic doubly orthogonal quasi-Sudoku Latin squares. In Figure 3 we construct $A_{1} \otimes A_{2}$ and $B_{1} \otimes B_{2}$ using the direct product

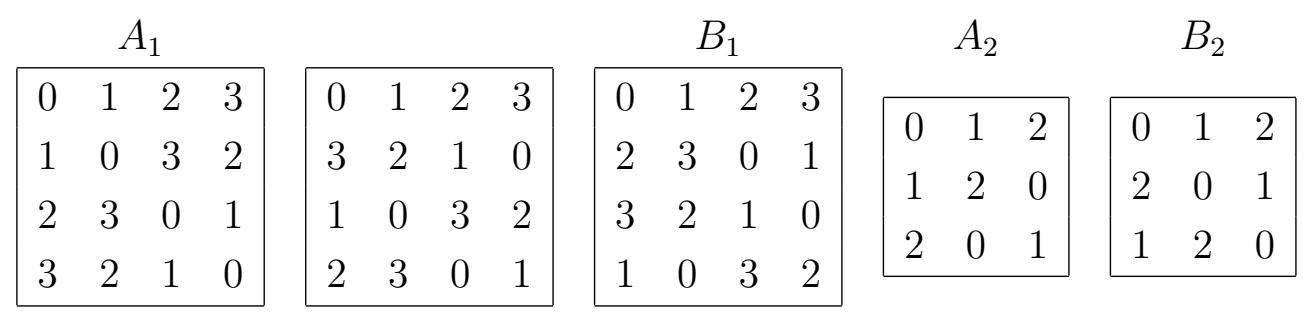

Figure 2: The three (pairwise) orthogonal Latin squares of order 4 and the two orthogonal Latin squares of order 3.

construction. To facilitate understanding, rows and columns have been labelled and $(x, y)$ has been replaced by $x y$. In Figure 4 the rows have been reordered as first $(p, 0)$ for $0 \leqslant p \leqslant 3$, then $(p, 1)$ for $0 \leqslant p \leqslant 3$, then $(p, 2)$ for $0 \leqslant p \leqslant 3$ to emphasise the fact that these squares are quasi-Sudoku Latin squares.

In Figure 5 we project down each subsquare in Figure 4 by applying the mappings $\Pi_{4}:[4] \times[3] \rightarrow[4]$ and $\Pi_{3}:[4] \times[3] \rightarrow[3]$, given by

$$
\begin{aligned}
& \Pi_{4}\left(\left(A_{1}(p, q), A_{2}(s, t)\right)\right)=\left(3 \times A_{1}(p, q)+A_{2}(s, t)\right) \bmod 4, \text { and } \\
& \Pi_{3}\left(\left(B_{1}(p, q), B_{2}(s, t)\right)\right)=\left(3 \times B_{1}(p, q)+B_{2}(s, t)\right) \bmod 3,
\end{aligned}
$$

to the entries in the quasi-Sudoku squares obtained by reordering the rows of the Latin squares $A_{1} \otimes A_{2}$ and $B_{1} \otimes B_{2}$ and label these as $\Pi_{4}\left(A_{1} \otimes A_{2}\right)$ and $\Pi_{3}\left(B_{1} \otimes B_{2}\right)$. Then, 
in Figure 6 we superimpose the projections $\Pi_{4}\left(A_{1} \otimes A_{2}\right)$ and $\Pi_{3}\left(B_{1} \otimes B_{2}\right)$ to verify that indeed $A_{1} \otimes A_{2}$ and $B_{1} \otimes B_{2}$ are doubly orthogonal quasi-Sudoku Latin squares of order 12 , as each $4 \times 3$ subsquare has each of the ordered pairs $(x, y), 0 \leqslant x \leqslant 3$ and $0 \leqslant y \leqslant 2$.

\begin{tabular}{|c|c|c|c|c|c|c|c|c|c|c|c|c|}
\hline \multicolumn{13}{|c|}{$A_{1} \otimes A_{2}$} \\
\hline & 00 & 01 & 02 & 10 & 11 & 12 & 20 & 21 & 22 & 30 & 31 & 32 \\
\hline 00 & 00 & 01 & 02 & 10 & 11 & 12 & 20 & 21 & 22 & 30 & 31 & 32 \\
\hline & 01 & 02 & 00 & 11 & 12 & 10 & 21 & 22 & 20 & 31 & 32 & 30 \\
\hline 0 & 02 & 00 & 01 & 12 & 10 & 11 & 22 & 20 & 21 & 32 & 30 & 31 \\
\hline 0 & 10 & 11 & 12 & 00 & 01 & 02 & 30 & 31 & 32 & 20 & 21 & 22 \\
\hline & 11 & 12 & 10 & 01 & 02 & 00 & 31 & 32 & 30 & 21 & 22 & 20 \\
\hline & 12 & 10 & 11 & 02 & 00 & 01 & 32 & 30 & 31 & 22 & 20 & 21 \\
\hline 0 & 20 & 21 & 22 & 30 & 31 & 32 & 00 & 01 & 02 & 10 & 11 & 12 \\
\hline & 21 & 22 & 20 & 31 & 32 & 30 & 01 & 02 & 00 & 11 & 12 & 10 \\
\hline & 22 & 20 & 21 & 32 & 30 & 31 & 02 & 00 & 01 & 12 & 10 & 11 \\
\hline & 30 & 31 & 32 & 20 & 21 & 22 & 10 & 11 & 12 & 00 & 01 & 02 \\
\hline & 31 & 32 & 30 & 21 & 22 & 20 & 11 & 12 & 10 & 01 & 02 & 00 \\
\hline & 32 & 30 & 31 & 22 & 20 & 21 & 12 & 10 & 11 & 02 & 00 & 01 \\
\hline
\end{tabular}

$$
B_{1} \otimes B_{2}
$$

\begin{tabular}{l|llllllllllll|}
\multicolumn{1}{l}{0} & 00 & 01 & 02 & 10 & 11 & 12 & 20 & 21 & 22 & 30 & 31 & 32 \\
\cline { 2 - 12 } 00 & 00 & 01 & 02 & 10 & 11 & 12 & 20 & 21 & 22 & 30 & 31 & 32 \\
01 & 02 & 00 & 01 & 12 & 10 & 11 & 22 & 20 & 21 & 32 & 30 & 31 \\
02 & 01 & 02 & 00 & 11 & 12 & 10 & 21 & 22 & 20 & 31 & 32 & 30 \\
\hline 10 & 20 & 21 & 22 & 30 & 31 & 32 & 00 & 01 & 02 & 10 & 11 & 12 \\
11 & 22 & 20 & 21 & 32 & 30 & 31 & 02 & 00 & 01 & 12 & 10 & 11 \\
12 & 21 & 22 & 20 & 31 & 32 & 30 & 01 & 02 & 00 & 11 & 12 & 10 \\
20 & 30 & 31 & 32 & 20 & 21 & 22 & 10 & 11 & 12 & 00 & 01 & 02 \\
21 & 32 & 30 & 31 & 22 & 20 & 21 & 12 & 10 & 11 & 02 & 00 & 01 \\
22 & 31 & 32 & 30 & 21 & 22 & 20 & 11 & 12 & 10 & 01 & 02 & 00 \\
30 & 10 & 11 & 12 & 00 & 01 & 02 & 30 & 31 & 32 & 20 & 21 & 22 \\
31 & 12 & 10 & 11 & 02 & 00 & 01 & 32 & 30 & 31 & 22 & 20 & 21 \\
32 & 11 & 12 & 10 & 01 & 02 & 00 & 31 & 32 & 30 & 21 & 22 & 20 \\
\cline { 2 - 9 } & & & & & & & & & & & &
\end{tabular}

Figure 3: A pair of orthogonal Latin squares of order 12 


\begin{tabular}{|c|c|c|c|c|c|c|c|c|c|c|c|c|}
\hline \multicolumn{13}{|c|}{ Reordered $A_{1} \otimes A_{2}$} \\
\hline & 00 & 01 & 02 & 10 & 11 & 12 & 20 & 21 & 22 & 30 & 31 & 32 \\
\hline 00 & 00 & 01 & 02 & 10 & 11 & 12 & 20 & 21 & 22 & 30 & 31 & 32 \\
\hline 10 & 10 & 11 & 12 & 00 & 01 & 02 & 30 & 31 & 32 & 20 & 21 & 22 \\
\hline 20 & 20 & 21 & 22 & 30 & 31 & 32 & 00 & 01 & 02 & 10 & 11 & 12 \\
\hline 30 & 30 & 31 & 32 & 20 & 21 & 22 & 10 & 11 & 12 & 00 & 01 & 02 \\
\hline 01 & 01 & 02 & 00 & 11 & 12 & 10 & 21 & 22 & 20 & 31 & 32 & 30 \\
\hline 11 & 11 & 12 & 10 & 01 & 02 & 00 & 31 & 32 & 30 & 21 & 22 & 20 \\
\hline 21 & 21 & 22 & 20 & 31 & 32 & 30 & 01 & 02 & 00 & 11 & 12 & 10 \\
\hline 31 & 31 & 32 & 30 & 21 & 22 & 20 & 11 & 12 & 10 & 01 & 02 & 00 \\
\hline 02 & 02 & 00 & 01 & 12 & 10 & 11 & 22 & 20 & 21 & 32 & 30 & 31 \\
\hline 12 & 12 & 10 & 11 & 02 & 00 & 01 & 32 & 30 & 31 & 22 & 20 & 21 \\
\hline 22 & 22 & 20 & 21 & 32 & 30 & 31 & 02 & 00 & 01 & 12 & 10 & 11 \\
\hline 32 & 32 & 30 & 31 & 22 & 20 & 21 & 12 & 10 & 11 & 02 & 00 & 01 \\
\hline
\end{tabular}

\begin{tabular}{|c|c|c|c|c|c|c|c|c|c|c|c|c|}
\hline \multicolumn{13}{|c|}{ Reordered $B_{1} \otimes B_{2}$} \\
\hline & 00 & 01 & 02 & 10 & 11 & 12 & 20 & 21 & 22 & 30 & 31 & 32 \\
\hline 00 & 00 & 01 & 02 & 10 & 11 & 12 & 20 & 21 & 22 & 30 & 31 & 32 \\
\hline 10 & 20 & 21 & 22 & 30 & 31 & 32 & 00 & 01 & 02 & 10 & 11 & 12 \\
\hline 20 & 30 & 31 & 32 & 20 & 21 & 22 & 10 & 11 & 12 & 00 & 01 & 02 \\
\hline 30 & 10 & 11 & 12 & 00 & 01 & 02 & 30 & 31 & 32 & 20 & 21 & 22 \\
\hline 01 & 02 & 00 & 01 & 12 & 10 & 11 & 22 & 20 & 21 & 32 & 30 & 31 \\
\hline 11 & 22 & 20 & 21 & 32 & 30 & 31 & 02 & 00 & 01 & 12 & 10 & 11 \\
\hline 21 & 32 & 30 & 31 & 22 & 20 & 21 & 12 & 10 & 11 & 02 & 00 & 01 \\
\hline 31 & 12 & 10 & 11 & 02 & 00 & 01 & 32 & 30 & 31 & 22 & 20 & 21 \\
\hline 02 & 01 & 02 & 00 & 11 & 12 & 10 & 21 & 22 & 20 & 31 & 32 & 30 \\
\hline 12 & 21 & 22 & 20 & 31 & 32 & 30 & 01 & 02 & 00 & 11 & 12 & 10 \\
\hline 22 & 31 & 32 & 30 & 21 & 22 & 20 & 11 & 12 & 10 & 01 & 02 & 00 \\
\hline 32 & 11 & 12 & 10 & 01 & 02 & 00 & 31 & 32 & 30 & 21 & 22 & 20 \\
\hline
\end{tabular}

Figure 4: A pair of orthogonal quasi-Sudoku Latin squares of order 12.

If the projections $\Pi_{4}$ and $\Pi_{3}$, given above, are replaced by the projections

$$
\begin{aligned}
& \Pi_{4}\left(\left(A_{1}(p, q), A_{2}(s, t)\right)\right)=A_{1}(p, q), \text { and } \\
& \Pi_{3}\left(\left(B_{1}(p, q), B_{2}(s, t)\right)\right)=B_{2}(s, t)
\end{aligned}
$$

we obtain the projected square given in Figure 7. Note that here each of the twelve subsquares can be obtained by reordering the rows and/or the columns of the first subsquare. 


\begin{tabular}{|c|c|c|c|c|c|c|c|c|c|c|c|c|}
\hline & & & & & $\Pi_{4}($ & $A_{1} \otimes$ & $\left.A_{2}\right)$ & & & & & \\
\hline & 00 & 01 & 02 & 10 & 11 & 12 & 20 & 21 & 22 & 30 & 31 & 32 \\
\hline 00 & 0 & 1 & 2 & 3 & 0 & 1 & 2 & 3 & 0 & 1 & 2 & 3 \\
\hline 10 & 3 & 0 & 1 & 0 & 1 & 2 & 1 & 2 & 3 & 2 & 3 & 0 \\
\hline 20 & 2 & 3 & 0 & 1 & 2 & 3 & 0 & 1 & 2 & 3 & 0 & 1 \\
\hline 30 & 1 & 2 & 3 & 2 & 3 & 0 & 3 & 0 & 1 & 0 & 1 & 2 \\
\hline 01 & 1 & 2 & 0 & 0 & 1 & 3 & 3 & 0 & 2 & 2 & 3 & 1 \\
\hline 11 & 0 & 1 & 3 & 1 & 2 & 0 & 2 & 3 & 1 & 3 & 0 & 2 \\
\hline 21 & 3 & 0 & 2 & 2 & 3 & 1 & 1 & 2 & 0 & 0 & 1 & 3 \\
\hline 31 & 2 & 3 & 1 & 3 & 0 & 2 & 0 & 1 & 3 & 1 & 2 & 0 \\
\hline 02 & 2 & 0 & 1 & 1 & 3 & 0 & 0 & 2 & 3 & 3 & 1 & 2 \\
\hline 12 & 1 & 3 & 0 & 2 & 0 & 1 & 3 & 1 & 2 & 0 & 2 & 3 \\
\hline 22 & 0 & 2 & 3 & 3 & 1 & 2 & 2 & 0 & 1 & 1 & 3 & 0 \\
\hline 32 & 3 & 1 & 2 & 0 & 2 & 3 & 1 & 3 & 0 & 2 & 0 & 1 \\
\hline
\end{tabular}

\begin{tabular}{|c|c|c|c|c|c|c|c|c|c|c|c|c|}
\hline \multicolumn{13}{|c|}{$\Pi_{3}\left(B_{1} \otimes B_{2}\right)$} \\
\hline & 00 & 01 & 02 & 10 & 11 & 12 & 20 & 21 & 22 & 30 & 31 & 32 \\
\hline 00 & 0 & 1 & 2 & 0 & 1 & 2 & 0 & 1 & 2 & 0 & 1 & 2 \\
\hline 10 & 0 & 1 & 2 & 0 & 1 & 2 & 0 & 1 & 2 & 0 & 1 & 2 \\
\hline 20 & 0 & 1 & 2 & 0 & 1 & 2 & 0 & 1 & 2 & 0 & 1 & 2 \\
\hline 30 & 0 & 1 & 2 & 0 & 1 & 2 & 0 & 1 & 2 & 0 & 1 & 2 \\
\hline 01 & 2 & 0 & 1 & 2 & 0 & 1 & 2 & 0 & 1 & 2 & 0 & 1 \\
\hline 11 & 2 & 0 & 1 & 2 & 0 & 1 & 2 & 0 & 1 & 2 & 0 & 1 \\
\hline 21 & 2 & 0 & 1 & 2 & 0 & 1 & 2 & 0 & 1 & 2 & 0 & 1 \\
\hline 31 & 2 & 0 & 1 & 2 & 0 & 1 & 2 & 0 & 1 & 2 & 0 & 1 \\
\hline 02 & 1 & 2 & 0 & 1 & 2 & 0 & 1 & 2 & 0 & 1 & 2 & 0 \\
\hline 12 & 1 & 2 & 0 & 1 & 2 & 0 & 1 & 2 & 0 & 1 & 2 & 0 \\
\hline 22 & 1 & 2 & 0 & 1 & 2 & 0 & 1 & 2 & 0 & 1 & 2 & 0 \\
\hline 32 & 1 & 2 & 0 & 1 & 2 & 0 & 1 & 2 & 0 & 1 & 2 & 0 \\
\hline
\end{tabular}

Figure 5: Orthogonal quasi-Sudoku Latin squares under projections $\Pi_{4}$ and $\Pi_{3}$.

This is not the case for the projected square given in Figure 6. To see this consider the first subsquare and any of the subsquares on rows 01,11,21 and 31 of Figure 6, setwise the rows of these subsquares do not equal any row in the first subsquare. 


\begin{tabular}{|c|c|c|c|c|c|c|c|c|c|c|c|c|}
\hline \multicolumn{13}{|c|}{$\Pi_{4}\left(A_{1} \otimes A_{2}\right), \Pi_{3}\left(B_{1} \otimes B_{2}\right)$} \\
\hline & 00 & 01 & 02 & 10 & 11 & 12 & 20 & 21 & 22 & 30 & 31 & 32 \\
\hline 00 & 0,0 & 1,1 & 2,2 & 3,0 & 0,1 & 1,2 & 2,0 & 3,1 & 0,2 & 1,0 & 2,1 & 3,2 \\
\hline 10 & 3,0 & 0,1 & 1,2 & 0,0 & 1,1 & 2,2 & 1,0 & 2,1 & 3,2 & 2,0 & 3,1 & 0,2 \\
\hline 20 & 2,0 & 3,1 & 0,2 & 1,0 & 2,1 & 3,2 & 0,0 & 1,1 & 2,2 & 3,0 & 0,1 & 1,2 \\
\hline 30 & 1,0 & 2,1 & 3,2 & 2,0 & 3,1 & 0,2 & 3,0 & 0,1 & 1,2 & 0,0 & 1,1 & 2,2 \\
\hline 01 & 1,2 & 2,0 & 0,1 & 0,2 & 1,0 & 3,1 & 3,2 & 0,0 & 2,1 & 2,2 & 3,0 & 1,1 \\
\hline 11 & 0,2 & 1,0 & 3,1 & 1,2 & 2,0 & 0,1 & 2,2 & 3,0 & 1,1 & 3,2 & 0,0 & 2,1 \\
\hline 21 & 3,2 & 0,0 & 2,1 & 2,2 & 3,0 & 1,1 & 1,2 & 2,0 & 0,1 & 0,2 & 1,0 & 3,1 \\
\hline 31 & 2,2 & 3,0 & 1,1 & 3,2 & 0,0 & 2,1 & 0,2 & 1,0 & 3,1 & 1,2 & 2,0 & 0,1 \\
\hline 02 & 2,1 & 0,2 & 1,0 & 1,1 & 3,2 & 0,0 & 0,1 & 2,2 & 3,0 & 3,1 & 1,2 & 2,0 \\
\hline 12 & 1,1 & 3,2 & 0,0 & 2,1 & 0,2 & 1,0 & 3,1 & 1,2 & 2,0 & 0,1 & 2,2 & 3,0 \\
\hline 22 & 0,1 & 2,2 & 3,0 & 3,1 & 1,2 & 2,0 & 2,1 & 0,2 & 1,0 & 1,1 & 3,2 & 0,0 \\
\hline 32 & 3,1 & 1,2 & 2,0 & 0,1 & 2,2 & 3,0 & 1,1 & 3,2 & 0,0 & 2,1 & 0,2 & 1,0 \\
\hline
\end{tabular}

Figure 6: The projected squares verifying that $A_{1} \otimes A_{2}$ and $B_{1} \otimes B_{2}$ are doubly orthogonal quasi-Sudoku Latin squares of order 12.

\section{Quasi-Sliced Orthogonal Arrays}

A (symmetric) orthogonal array, denoted $O A(N, k, s, t)$, is an $N \times k$ array with entries chosen from the set $[s]$ of levels such that for every $N \times t$ submatrix the $s^{t}$ level combinations of $\overbrace{[s] \times \cdots \times[s]}^{t}$ each occur a constant number of times. We define a sliced symmetric orthogonal array to be an $O A(N, k, s, t), \mathcal{O}$, which satisfies the following property:

- there exists a projection $\Pi$ from $[s]$ to $\left[s_{0}\right], s_{0}<s$, and a partition of the rows of $\mathcal{O}$ into $\nu$ subarrays, $\mathcal{O}_{i}$, such that when the $s$ levels of $[s]$ are collapsed according to the projection $\Pi$ each $\mathcal{O}_{i}$ forms a symmetric $O A\left(N_{0}, k, s_{0}, t\right)$.

We say $\left(\mathcal{O}_{1}, \mathcal{O}_{2}, \ldots, \mathcal{O}_{\nu}\right)$ is a sliced symmetric orthogonal array.

For a $k$ factor design, let $S=\left\{s_{1}, \ldots, s_{k}\right\}$ denote the list of numbers of factor levels. An asymmetric orthogonal array, denoted $O A(N, k, S, t)$, is an $N \times k$ array, where the $j$ th column contains entries of $\left[s_{j}\right], s_{j} \in S$, and for each $t$-subset $T$ of columns, the $N \times t$ submatrix defined by these columns contains all $\prod_{i \in T} s_{i}$ tuples of $\prod_{i \in T}\left[s_{i}\right]$ (the Cartesian product of $\left.\left[s_{i}\right], i \in T\right)$ a constant, $\lambda_{T}$, number of times. We define a quasi-sliced asymmetric orthogonal array to be an $O A(N, k, S, t)$ array, $\mathcal{O}$, which satisfies the following property: 


\begin{tabular}{|c|c|c|c|c|c|c|c|c|c|c|c|c|}
\hline \multicolumn{13}{|c|}{$\Pi_{4}\left(A_{1} \otimes A_{2}\right), \Pi_{3}\left(B_{1} \otimes B_{2}\right)$} \\
\hline & 00 & 01 & 02 & 10 & 11 & 12 & 20 & 21 & 22 & 30 & 31 & 32 \\
\hline 00 & 0,0 & 0,1 & 0,2 & 1,0 & 1,1 & 1,2 & 2,0 & 2,1 & 2,2 & 3,0 & 3,1 & 3,2 \\
\hline 10 & 1,0 & 1,1 & 1,2 & 0,0 & 0,1 & 0,2 & 3,0 & 3,1 & 3,2 & 2,0 & 2,1 & 2,2 \\
\hline 20 & 2,0 & 2,1 & 2,2 & 3,0 & 3,1 & 3,2 & 0,0 & 0,1 & 0,2 & 1,0 & 1,1 & 1,2 \\
\hline 30 & 3,0 & 3,1 & 3,2 & 2,0 & 2,1 & 2,2 & 1,0 & 1,1 & 1,2 & 0,0 & 0,1 & 0,2 \\
\hline 01 & 0,2 & 0,0 & 0,1 & 1,2 & 1,0 & 1,1 & 2,2 & 2,0 & 2,1 & 3,2 & 3,0 & 3,1 \\
\hline 11 & 1,2 & 1,0 & 1,1 & 0,2 & 0,0 & 0,1 & 3,2 & 3,0 & 3,1 & 2,2 & 2,0 & 2,1 \\
\hline 21 & 2,2 & 2,0 & 2,1 & 3,2 & 3,0 & 3,1 & 0,2 & 0,0 & 0,1 & 1,2 & 1,0 & 1,1 \\
\hline 31 & 3,2 & 3,0 & 3,1 & 2,2 & 2,0 & 2,1 & 1,2 & 1,0 & 1,1 & 0,2 & 0,0 & 0,1 \\
\hline 02 & 0,1 & 0,2 & 0,0 & 1,1 & 1,2 & 1,0 & 2,1 & 2,2 & 2,0 & 3,1 & 3,2 & 3,0 \\
\hline 12 & 1,1 & 1,2 & 1,0 & 0,1 & 0,2 & 0,0 & 3,1 & 3,2 & 3,0 & 2,1 & 2,2 & 2,0 \\
\hline 22 & 2,1 & 2,2 & 2,0 & 3,1 & 3,2 & 3,0 & 0,1 & 0,2 & 0,0 & 1,1 & 1,2 & 1,0 \\
\hline 32 & 3,1 & 3,2 & 3,0 & 2,1 & 2,2 & 2,0 & 1,1 & 1,2 & 1,0 & 0,1 & 0,2 & 0,0 \\
\hline
\end{tabular}

Figure 7: The projected squares verifying that $A_{1} \otimes A_{2}$ and $B_{1} \otimes B_{2}$ are doubly orthogonal quasi-Sudoku Latin squares of order 12.

- there exist projections $\Pi_{i}:\left[s_{i}\right] \rightarrow\left[s_{i}^{\prime}\right]$ for $i=1, \ldots, k$ and a partition of the rows $\mathcal{O}$ into $\nu$ subarrays, $\mathcal{O}_{i}$, such that when the sets of levels $\left[s_{1}\right], \ldots,\left[s_{k}\right]$ are respectively collapsed onto $\left[s_{1}^{\prime}\right], \ldots,\left[s_{k}^{\prime}\right], s_{i}^{\prime}<s_{i}$, each $\mathcal{O}_{i}$ is an asymmetric $O A\left(N_{0}, k, S^{\prime}, t\right)$, $S^{\prime}=\left\{s_{1}^{\prime}, s_{2}^{\prime}, \ldots, s_{k}^{\prime}\right\}$.

More precisely $\left(\mathcal{O}_{1}, \ldots, \mathcal{O}_{\nu}\right)$ is said to be a quasi-sliced asymmetric orthogonal array. In the above definition we note that if each projections is not one-to-one then it is immediate that $s_{i}^{\prime}<s_{i}$, for all $i$.

Proposition 5.1. If $A_{1}$ and $B_{1}$ are pairwise orthogonal Latin squares of order $m$ and $A_{2}$ and $B_{2}$ are pairwise orthogonal Latin squares of order $n$. Then there exists a quasi-sliced asymmetric orthogonal array $O A\left(m^{2} n^{2}, 4,\{m n\}, 2\right)$.

Proof. "Unstack" the pair of orthogonal quasi-Sudoku Latin squares $A_{1} \otimes A_{2}$ and $B_{1} \otimes B_{2}$ to obtain an orthogonal array $O A\left(m^{2} n^{2}, 4,\{m n\}, 2\right), \mathcal{O}$, where row $(r, c)$ of $\mathcal{O}$ takes the form

$$
\left[r, \quad c, \quad\left(A_{1} \otimes A_{2}\right)(r, c), \quad\left(B_{1} \otimes B_{2}\right)(r, c)\right] .
$$

To verify that this is a quasi-sliced asymmetric orthogonal array we first provide details of the partitioning of the rows and then the projections. 
Recall that the rows of the reordered squares $A_{1} \otimes A_{2}$ and $B_{1} \otimes B_{2}$ are labelled as $(p, s)$, $0 \leqslant p \leqslant m-1$ and $0 \leqslant s \leqslant n-1$ and the columns are labelled as $(q, t), 0 \leqslant q \leqslant m-1$ and $0 \leqslant t \leqslant n-1$. Now for fixed $q$ let $\mathcal{O}_{q}$ be the $\left(m n^{2}\right) \times 4$ subarray with rows indexed by $((p, s),(q, t)), 0 \leqslant p \leqslant m-1$ and $0 \leqslant s, t \leqslant n-1$, where a row takes the form

$$
\left[(p, s), \quad(q, t), \quad\left(A_{1} \otimes A_{2}\right)((p, s),(q, t)),\left(B_{1} \otimes B_{2}\right)((p, s),(q, t))\right] .
$$

Focusing on the rows corresponding to the subarray $\mathcal{O}_{q}$, before we apply the projections $\Pi_{1}, \Pi_{2}, \Pi_{3}, \Pi_{4}$, we see that for columns 1,3 and 4 the set of levels is $\{(p, s) \mid 0 \leqslant p \leqslant$ $m-1,0 \leqslant s \leqslant n-1\}$, for column 2 the set of levels is $\{(q, t) \mid 0 \leqslant t \leqslant n-1\}$. Next apply the projections

$$
\begin{aligned}
& \Pi_{1}:(p, s) \rightarrow s \\
& \Pi_{2}:(q, t) \rightarrow t \\
& \Pi_{3}=\Pi_{m} \\
& \Pi_{4}=\Pi_{n}
\end{aligned}
$$

where $\Pi_{m}$ and $\Pi_{n}$ are defined in the proof of Proposition 3.2. Note that each of these projections is not one-to-one.

Finally we need to prove that $\mathcal{O}_{q}$ is a asymmetric orthogonal array with parameters $O A\left(m n^{2}, 4, S^{\prime}, 2\right)$ where $S^{\prime}=\{n, n, m, n\}$.

Note columns 3 and 4 of $\mathcal{O}_{q}$, are the concatenation of the $n$ sets of pairs

$$
\left\{\left(\Pi_{m}\left(A_{1}(p, q), A_{2}(s, t)\right), \Pi_{n}\left(B_{1}(p, q), B_{2}(s, t)\right)\right) \mid 0 \leqslant p \leqslant m-1,0 \leqslant t \leqslant n-1\right\}
$$

one set for each $0 \leqslant s \leqslant n-1$. The set $\left\{\left(\Pi_{m}\left(A_{1}(p, q), A_{2}(s, t)\right), \Pi_{n}\left(\left(B_{1}(p, q), B_{2}(s, t)\right)\right) \mid\right.\right.$ $0 \leqslant p \leqslant m-1,0 \leqslant t \leqslant n-1\}=[m] \times[n]$. Hence columns 3 and 4 are the concatenation of $n$ copies of $[m] \times[n]$.

For fixed $s$ the set of pairs $\left\{\left(s, \Pi_{m}\left(A_{1}(p, q), A_{2}(s, t)\right)\right) \mid 0 \leqslant p \leqslant m-1,0 \leqslant t \leqslant n-1\right\}$ gives $n$ copies of $\{s\} \times[m]$ and $\left\{\left(s,\left(\Pi_{n}\left(B_{1}(p, q), B_{2}(s, t)\right)\right) \mid 0 \leqslant p \leqslant m-1,0 \leqslant t \leqslant n\right\}\right.$ gives $m$ copies of $\{s\} \times[n]$. So, respectively, as $s$ takes the values $0, \ldots, n-1$ we obtain $n$ copies of $[n] \times[m]$ and $m$ copies of $[n] \times[n]$.

For fixed $s$ the set $\left\{\left(t, \Pi_{m}\left(A_{1}(p, q), A_{2}(s, t)\right)\right) \mid 0 \leqslant p \leqslant m-1,0 \leqslant t \leqslant n-1\right\}$ gives one copy of $[n] \times[m]$. So as $s$ takes the values $0, \ldots, n-1$ we obtain $n$ copies of $[n] \times[m]$. Finally as $s$ takes the values $0, \ldots, n-1\left\{\left(t, \Pi_{n}\left(B_{1}(p, q), B_{2}(s, t)\right)\right) \mid 0 \leqslant p \leqslant m-1,0 \leqslant t \leqslant n-1\right\}$ gives $m$ copies of $[n] \times[n]$.

Thus each $\mathcal{O}_{q}$, for $0 \leqslant q \leqslant m-1$ is an asymmetric orthogonal array with the required parameters. In all cases the projections are not one-to-one and the results is a quasi-sliced asymmetric orthogonal array as required. 
Once again, for $m=n$, the extension of these pairwise properties to $K>2$ orthogonal direct product designs $O A\left(m^{2} n^{2}, 2+K,\{m n\}, 2\right)$ is immediate, if the component designs are available.

By way of example we project the first three columns of the array given in Figure 6 to obtain the quasi orthogonal array $\mathcal{O}_{0}(q=0)$ with parameters $O A\left(36,4, S^{\prime}, 2\right)$, where $S^{\prime}=\{3,3,4,3\}$.

$\mathcal{O}_{0}$

000000000000111111111111222222222222

012012012012012012012012012012012012

012301230123120013302231201130023312

012012012012201201201201120120120120

Figure 8: The projected sliced array $\mathcal{O}_{0}$

\section{Construction of Quasi-Sudoku-Based Sliced Space- Filling Designs}

Sliced space-filling designs can be constucted from the doubly orthogonal quasi-Sudoku Latin squares or the quasi-sliced asymmetic orthogonal array using the techniques in [5], [7], and [9]. In brief, the column-wise procedure is as follows. First, randomly relabel each column's elements $1, \ldots, m n$ subject to the constraint that the elements mapped to the same symbol by $\Pi$ form a consecutive subset of $1, \ldots, m n$. Next, replace the elements in each column with symbol $k$ with a random permutation of $(k-1) m n+$ $1, \ldots,(k-1) m n+m n$. Lastly, each element of the space-filling is generated as $\left(x_{i j}-\right.$ $\left.u_{i j}\right) /(m n)^{2}$, where $x_{i j}$ denotes the design elements after the first two steps and $u_{i j}$ denotes a random Uniform $(0,1)$ deviate. As an example, Figure 9 shows a sliced space-filling design constructed based on the quasi-sliced asymmetic orthogonal array whose first sliced is shown in Figure 8.

As shown in [5] and [9], the complete design achieves uniformity in one and twodimensional projections, while the slices are guaranteed to achieve uniformity in twodimensional projections. On the other hand, the columns based on the doubly orthogonal quasi-Sudoku Latin squares can be divided into a larger number of slices, as defined by the quasi-Sudoku sub-squares, each of which has guaranteed uniformity under one and two-dimensional projections, as shown in [9]. 


\begin{tabular}{|c|c|c|c|}
\hline V & 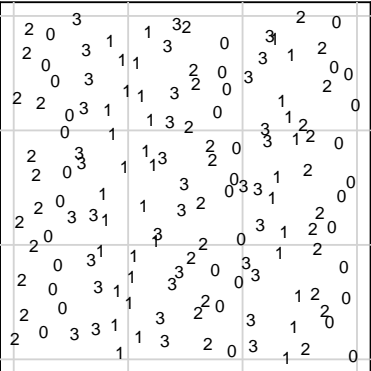 & 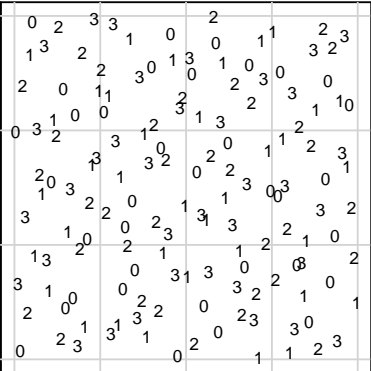 & 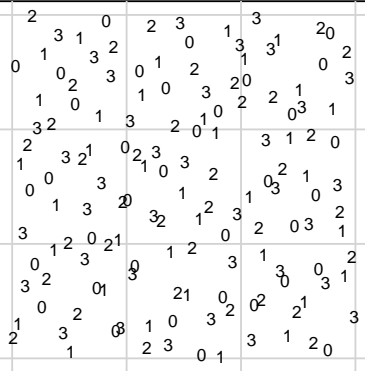 \\
\hline 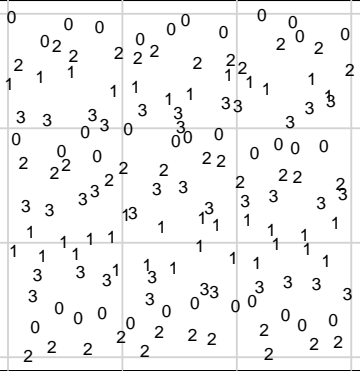 & Variable 2 & 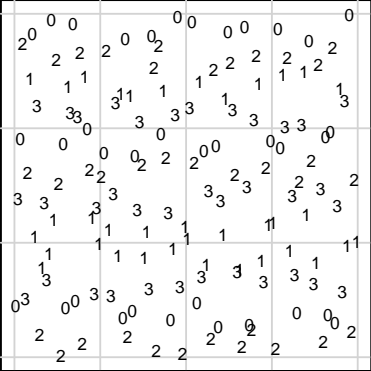 & 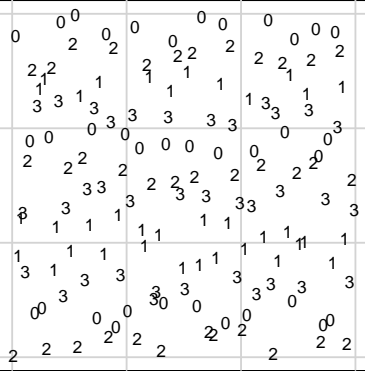 \\
\hline 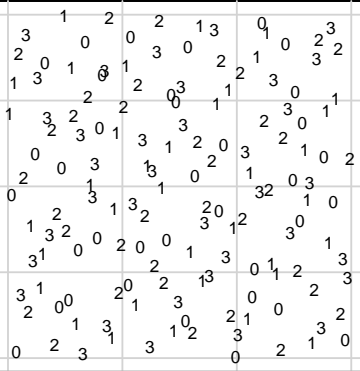 & 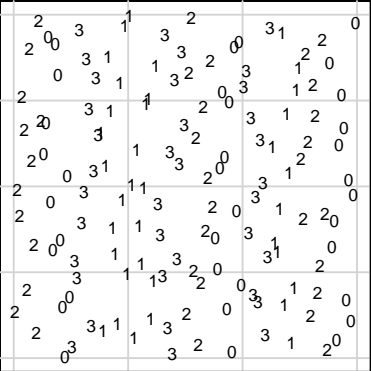 & Variable 3 & 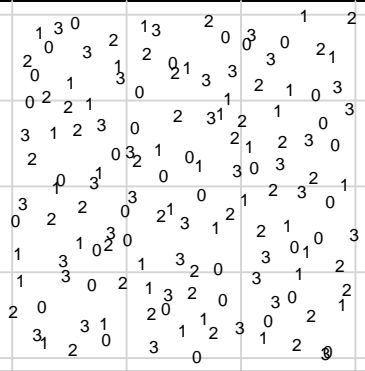 \\
\hline 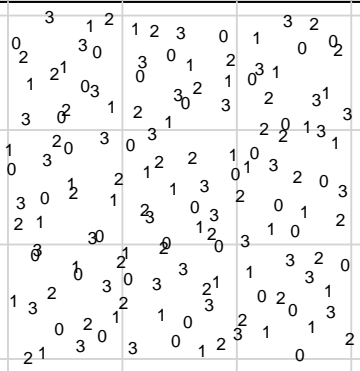 & 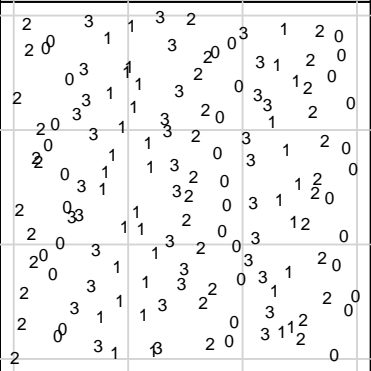 & 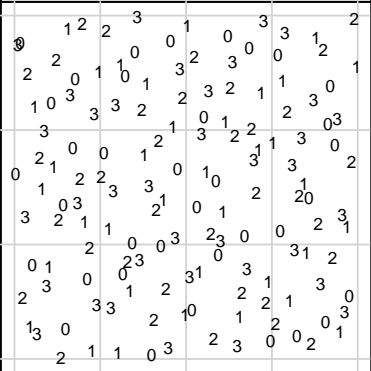 & Variable 4 \\
\hline
\end{tabular}

Figure 9: Sliced space-filling design based on a quasi-sliced asymmetic orthogonal array. Slices indicated by point labels.

\section{References}

[1] Colbourn, C. J., Dinitz, J. H. (Eds.). (2010). Handbook of combinatorial designs. CRC press.

[2] Dénes, J., Keedwell, A.D. (1974). Latin Squares and their Applications. London: English Universities Press limited.

[3] Haaland, B., Qian, P. Z. (2010). An approach to constructing nested space-filling designs for multi-fidelity computer experiments. Statistica Sinica, 20(3), 1063. 
[4] Pedersen, R. M., Vis, T. L. (2009). Sets of mutually orthogonal Sudoku Latin squares. The College Mathematics Journal, 40(3), 174-181.

[5] Qian, P. Z., Wu, C. J. (2009). Sliced space-filling designs. Biometrika, 96(4), 945-956.

[6] Raghavarao, D. (1988). Constructions and Combinatorial Problems in Design of Experiments (corrected reprint of the 1971 Wiley ed.). New York: Dover.

[7] Tang, B. (1993). Orthogonal array-based Latin hypercubes. Journal of the American Statistical Association, 88(424), 1392-1397.

[8] http://puzzles.usatoday.com/sudoku/ [accessed: 19 September 2013]

[9] Xu, X., Haaland, B., Qian, P. Z. (2011). Sudoku-based space-filling designs. Biometrika, 98(3), 711-720.

[10] Zhang, Q., Qian, P. Z. (2013). Designs for crossvalidating approximation models. Biometrika, ast034. 